\title{
Identification and Quantification of Natural Lecithin Phospholipids and Their Residuals in Freeze-Dried and Drum-Dried Fruits and Vegetables by LC-MS and HPLC-ELSD
}

\author{
Xiaoyan $\mathrm{Xia}^{1}$, Tiffany Gallegos-Peretz ${ }^{1}$ \& Boris V. Nemzer ${ }^{1,2}$ \\ ${ }^{1}$ VDF FutureCeuticals, Inc., Momence, IL, USA \\ ${ }^{2}$ Department of Food Science and Human Nutrition, University Illinois at Urbana Champaign, Urbana, IL, USA \\ Correspondence: Boris Nemzer. FutureCeuticals, Inc. 2692 N. State Rt. 1-17, Momence, IL, 60954, USA. Tel: \\ 1-812-507-1427. Email: bnemzer@futureceuticals.com
}

Received: March 15, 2017

doi:10.5539/jfr.v6n3p116
Accepted: May 4, 2017 Online Published: May 12, 2017

URL: https://doi.org/10.5539/jfr.v6n3p116

\begin{abstract}
Sunflower lecithin is commonly used as a food processing agent. In this study, residues of sunflower lecithin phospholipids in drum-dried fruits and vegetables were investigated. The contents of phosphatidylcholine and phosphatidylethanolamine were of interest due to their natural levels in fresh fruits and vegetables as well as their residues after the drum drying process. Identification of these compounds in freeze-dried and drum-dried fruits and vegetables was conducted by normal-phase and reverse-phase ultra-high-performance liquid chromatography (UPLC) coupled with Q Exactive Orbitrap electrospray mass spectrometry. Quantification of phosphatidylcholine in various fruits and vegetables was performed using normal-phase high-performance liquid chromatography with evaporative light scattering detector (ELSD). The quantification results from these various products demonstrate that use of de-oiled sunflower lecithin as a processing agent in the drum drying production process does not affect the quality of final drum-dried products.
\end{abstract}

Keywords: phospholipid, phosphatidylcholine, phosphatidylethanolamine, liquid chromatography, mass spectrometer, HPLC-ELSD

\section{Introduction}

Lecithin, a natural complex mixture of different phospholipids, is extensively used in the food processing, pharmaceutical, cosmetics and paint industries. Frequently-used lecithins in food processing include soybean, canola, and sunflower lecithin. The major compounds of phospholipid in edible fats are phosphatidylcholine (PC), phosphatidylinositol (PI), phosphatidylserine (PS) and phosphatidylethanolamine (PE) (Fig. 1) (E. O. Aluyor, Ozigagu, Oboh \& P. Aluyor, 2009).

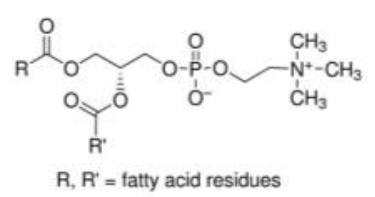

L- $\alpha$-Phosphatidylcholine

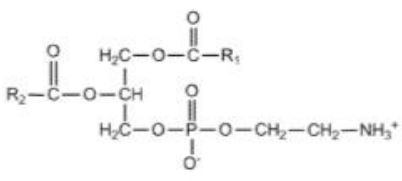

$\mathrm{R} 1, \mathrm{R} 2=$ fatty acid residues

L- $\alpha$-phosphatidylethanolamine

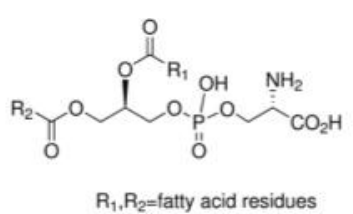

L- $\alpha$-phosphatidyl-L-serine

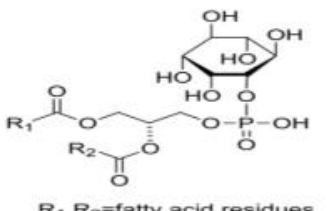

L- $\alpha$-phosphatidylinositol

Figure 1. The chemical structures of typical lecithin phospholipids 
Lecithin is an important processing agent in the drum drying production process due to its emulsifying properties. Fruits and vegetables, especially ones with high sugar content, become difficult to remove from drums at high temperatures (Tang, Feng \& Shen, 2003; Pua, Hamid, Rusul \& Rahman, 2007). Consequently, de-oiled sunflower lecithin is often added during production process to facilitate removal of the final dried product. Due to the relative scarcity of non-genetically modified soybeans, sunflower lecithin is becoming more widely used in various food production processes.

In order to evaluate any residual effect of de-oiled sunflower lecithin phospholipids in drum dried products, lecithin analysis must be conducted in both: a.) the raw material used for drum drying; and, b.) the corresponding final product. With regards to lecithin analysis, several methods have historically been used by researchers in order to identify and quantify phospholipids in different sample matrices (Krüger, Bürmannm \& Morlock, 2015; Carelli, Brevedan \& Crapiste, 1997; Sparace \& Moore, 1979; Block, Borne, Joyard \& Douce, 1983; Dorne, Joyard, Block \& Douce, 1985; Jangle, Galge, Patil \& Thorat, 2013; Jungalwala, Evans \& McCluer, 1984; McDonald, Robin \& Siegel, 1981; Prabha, Raina \& Patwardhan, 1988; Robins \& Patton, 1986). Thin-layer chromatography, high performance liquid chromatography and nuclear magnetic resonance spectroscopy have been used to analyze phospholipids (Helmerich \& Koehler, 2003). Detectors such as refractive index (RI), ultraviolet (UV), mass spectrometry and evaporative light scattering detectors have been used to test for phospholipids (Picchioni, Watada \& Whitaker, 1996; Letter, 1992; Becart, Chevalier \& Blesse, 1990). However, RI detectors have shown low sensitivity and a high degree of variability (depending upon the solvents used). UV detectors do not allow for the use of mobile phase that absorbs in UV range, such as chloroform that has been widely used in normal phase systems (Descalzo, Insani \& Pensel, 2003). In order to avoid the undesirable baseline drift caused by solvent gradients during UV analyses, and to better facilitate effective separation and flexible gradient elution by high performance liquid chromatography, we used UHPLC coupled with Q Exactive Orbitrap mass spectrometry to identify all detected phospholipids, and HPLC combined with ELSD detector to quantify total PC content in various fruits and vegetables (especially due to the fact that phospholipid content for most of those samples are unavailable in the literature). At the same time, PC content in corresponding drum dried products was also tested to elucidate any de-oiled sunflower lecithin residue present after the drum drying production process.

The main objective of this work was to develop a quick, economical procedure to quantify phospholipid classes present in drum-dried fruits and vegetables across a wide range of concentrations. The developed method was applied to study the residues of de-oiled sunflower lecithin phospholipids that may remain after the drum drying process. The differences of lecithin phospholipids content between raw material and final drum-dried product were determined and ultimately used as a quality control factor during the production process.

\section{Materials and Methods}

\subsection{Chemicals and Reagents}

Omnisolv ${ }^{\circledR}$ grade of methanol, chloroform from EMD were purchased from VWR (Radnor, PA, USA). Isopropanol was obtained from Burdick \& Jackson (Muskegon, MI, USA). HPLC grade of hexane was from Sigma-Aldrich (St. Louis, MO, USA). Ammonium hydroxide from BDH ARISTAR was purchased from VWR (Radnor, PA, USA) and water was purified using a Millipore Q-POD element unit (passed through a $0.22 \mu \mathrm{m}$ milli pak ${ }^{\circledR}$ express filter to produce $18.2 \mathrm{M} \Omega . \mathrm{cm}$ resistivity). The L- $\alpha$-phosphatidylcholine, L- $\alpha$-phosphatidylethanolamine, L- $\alpha$-phosphatidyl-L-serine and L- $\alpha$-phosphatidylinositol phospholipid standards were purchased from Sigma-Aldrich (St. Louis, MO, USA). The freeze dried product and drum dried product of spinach, cranberry, cherry, blueberry, apple, pineapple, sweet potato, quinoa, pumpkin and broccoli sprout powder were provided by Van Drunen Farms (Momence, IL, USA).

\subsection{Sample and Standard Preparation}

About $1 \mathrm{~g}$ of sample was weighed and quantitatively transferred to a $50 \mathrm{~mL}$ centrifuge tube. $10 \mathrm{~mL}$ of chloroform and methanol mixture at a ratio of $6: 4$ respectively was then added to the centrifuge tube. The sample was briefly vortexed and then sonicated for $10 \mathrm{mins}$, shaken for 10mins, and centrifuged at 9,000 rpm $(13843 \mathrm{x} \mathrm{g}) 20^{\circ} \mathrm{C}$ for $10 \mathrm{mins}$. After centrifugation, $2.5 \mathrm{~mL}$ of supernatant was transferred to another $50 \mathrm{~mL}$ centrifuge tube and $0.5 \mathrm{~mL}$ of DI water was added to re-extract the sample. The sample was again centrifuged at 9,000 rpm (13843 x g) $20^{\circ} \mathrm{C}$ for $10 \mathrm{mins}$. After centrifugation, the bottom layer was extracted and dried with nitrogen. Then the sample was reconstituted with $2.5 \mathrm{~mL}$ of chloroform and methanol mixture, again in the ratio of $6: 4$. The reconstituted sample was filtered with a $0.2 \mu \mathrm{m}$ PTFE filter (Pall Corporation) into an auto sampler vial for HPLC analysis. All of the samples were prepared and analyzed for triplicate. The results were expressed as the mean of triplicates. Stock solutions of the phospholipid standards were prepared in chloroform/methanol $(6: 4, \mathrm{v} / \mathrm{v})$ and 
diluted to different concentrations.

\subsection{LC-MS Analysis}

Thermo Scientific's ultimate 3000 UHPLC coupled with Q Exactive Orbitrap mass spectrometry (Thermo Fisher Scientific, Bremen, Germany) was used to perform phospholipid identification analysis. The UHPLC system consists of Ultimate 3000 binary pump, autosampler, column compartment and diode array detector. The system was controlled by Xcalibur 4.0 software. The separation of phospholipids was first carried out on Thermo Accucore C18 column by an isocratic elution with isopropyl alcohol, DI water and methanol in the proportion of 70:22:8 (v/v) to obtain the fingerprints of PC and PE respectively. The process was then conducted on a Luna ${ }^{\circledR}$ $3 \mu \mathrm{m}$ silica 100 $250 \times 2 \mathrm{~mm}$ LC column from Phenomenex by an isocratic elution with isopropyl alcohol, hexane, and DI water in the ratio of 50:40:10 (v/v) with $0.1 \%$ of formic acid to separate different phospholipids. The mobile phase flow rate was $200 \mu \mathrm{L} \mathrm{min}{ }^{-1}$. The sample injection volume was $2 \mu \mathrm{L}$ and the total run time is 35 mins.

A heated electrospray ionization (HESI) source was installed in the Q Exactive mass spectrometer. The optimized capillary temperature was set at $250{ }^{\circ} \mathrm{C}$; spray voltage $3.5 \mathrm{kv}$, sheath gas flow rate 35 arbitrary unit, auxiliary gas flow rate 10 arbitrary unit, and auxiliary gas heater temperature $350{ }^{\circ} \mathrm{C}$. All of the data in this study were acquired using full MS scan mode and data dependent acquisition mode using positive polarity. With the full scan, the mass analyzer was scanned from the first mass $\mathrm{m} / \mathrm{z} 150$ to the last mass $\mathrm{m} / \mathrm{z} 1000$ without interruption. The mass resolution of the mass spectrometer was set at 70000 FWHM (Full width at half maximum, $\mathrm{m} / \mathrm{z} 200$ ) and AGC (Automatic gain control) target at 1e6. The phospholipid standard solution was first run in full MS scan to obtain the precursor ions of different phospholipids. In the dd-MS ${ }^{2}$ experiment, the mass spectrometer performed data-independent acquisition, and covered the scan range by targeted HCD (higher energy collisional dissociation) events with isolation windows defined in the inclusion list for confirmation analysis. The targeted precursor ions were included in the inclusion list. The precursor ions were selected by quadrupole and sent to the HCD collision cell of the Q Orbitrap and fragmented with normalized collision energy to yield the product ions. The mass resolution of the mass spectrometer was set at 70000 FWHM and NCE (normalized fragmentation energy) 30\%. AGC target was set at $1 \mathrm{e} 6$ and isolation window at $2 \mathrm{~m} / \mathrm{z}$.

\subsection{HPLC-ELSD Analysis}

The Agilent HPLC series 1100 (Agilent Technologies company, Santa Clara, CA, USA) was used to conduct the phospholipid quantification analysis. The HPLC system consists of Agilent 1100 micro vacuum degasser, a quaternary pump system, and a thermostated column compartment. Agilent 1260 Infinity ELSD detector was connected to the Agilent system by Agilent $95900 \mathrm{E}$ dual channel interface module which converted the analog ELSD signal to digital data that could be processed by Chemstation CDS software. A Luna $5 \mu \mathrm{m}$ silica $100 \AA$ 250x4.6mm LC column from Phenomenex (Phenomenex, Torrance, CA, USA) was used to conduct quantification analysis. Separations were performed at $30^{\circ} \mathrm{C}$. Initially, a hexane/isopropanol/water mixture that had been used extensively was applied in our HPLC system for phospholipid analysis. However, the resulting base line was very noisy. In order to address this issue, the mobile phases were switched to chloroform, methanol, water and ammonium hydroxide and a multistep elution gradient method was applied to elute the standard compounds and to analyze samples. The gradient profile (Table 1) was as such: $100 \%$ of chloroform was used as solvent A; $100 \%$ of methanol as solvent B; $100 \%$ of DI water as solvent C; $0.14 \%$ of ammonium hydroxide as solvent $\mathrm{D}$. The gradient profile was $0-15 \mathrm{~min}$ from $23 \%$ to $34 \% \mathrm{~B}$; $15-25 \mathrm{~min}$ from $34 \%$ to $40 \% \mathrm{~B} ; 25-25.1 \mathrm{~min}$ from $40 \%$ to $49 \% \mathrm{~B}$, and kept to $30 \mathrm{~min}$. Gradient polarity was increased by addition of methanol. The separation of PE, PS, PI and PC was achieved within 20 mins. At $30.1 \mathrm{~min}$, the mobile phases were back to initial composition and continued to run for $15 \mathrm{mins}$ to equilibrate the system before another injection. The mobile phase flow rate was $1.0 \mathrm{~mL} \mathrm{~min}{ }^{-1}$. The total chromatographic run time was $45 \mathrm{mins}$ per sample. The nebulizing temperature of ELSD detector was set up at $60^{\circ} \mathrm{C}$ to evaporate the mobile phase in the nebulizer. The nitrogen pressure was set at 4.0 bar.

\subsection{Statistical Analysis}

Data are presented as mean values with the standard errors of the means. The data set was tested for normality. For multiple comparison, one-way ANOVA was used. Statistical significance was set at $\mathrm{P} \leq 0.05$. Statistical analysis was done with SigmaPlot 10 (Systat Software, Inc). 
Table 1. The solvent gradients used in HPLC-ELSD analysis (Percentage)

\begin{tabular}{ccccc}
\hline Time (min) & Chloroform & Methanol & Water & Ammonium hydroxide $(0.14 \%)$ \\
\hline 0 & 75.5 & 23 & 1 & 0.5 \\
15 & 60 & 34 & 5.5 & 0.5 \\
25 & 53.5 & 40 & 6 & 0.5 \\
25.1 & 0 & 49 & 50.5 & 0.5 \\
30 & 0 & 49 & 50.5 & 0.5 \\
30.1 & 75.5 & 23 & 1 & 0.5 \\
45 & 75.5 & 23 & 1 & 0.5 \\
\hline
\end{tabular}

\section{Results and Discussion}

\subsection{Characterization of PC and PE by UHPLC-ESI ${ }^{+}-M S$ in Different Natural Products}

Typically, PC and PE are the major phospholipid classes in sunflower lecithin (Krüger, Bürmann \& Morlock, 2015). Thus, identification analysis focused on these two compounds even though all of the four phospholipid classes were screened in each sample tested. Since it is impossible to distinguish isobaric peaks that have the same nominal $\mathrm{m} / \mathrm{z}$ values of different chemical structures or structural isomers of a specific compound solely based on the mass spectra (Stübiger, Pittenauer, Belgacem, Rehulka, Widhalm \& Allmaier, 2009), we combined retention time and mass spectra of authentic standards for identification analysis. To characterize PC and PE, their authentic standards were first analyzed using liquid chromatography coupled with Q Exactive Orbitrap mass spectrometry on positive mode to obtain the retention time and mass spectra of typical lipid compounds. De-oiled sunflower lecithin and a variety of fruits and vegetables were then analyzed using the same methodology. Both normal phase and reverse phase columns were used to conduct separation. Full MS scan and data dependent MS2 were used to obtain the product ions of target compounds.

$\mathrm{PE}$ and PC in de-oiled sunflower lecithin were separated into two different peaks by normal phase LC/MS (Fig. 2). The species of phospholipid within the same class could not be separated with each other solely using chromatography. However, we were able to differentiate them by their corresponding mass spectra.

The mass spectra of PC in de-oiled sunflower lecithin samples are shown on Figure 2. m/z 758.56830 and m/z 782.56812 were the dominant molecular species. The corresponding sodium adducts were observed at $\mathrm{m} / \mathrm{z}$ 780.55096 and $\mathrm{m} / \mathrm{z}$ 804.55005. This indicated the presence of PC 34:2 and PC 36:4. These results are consistent with the literature (Krüger, Bürmann \& Morlock, 2015), in which sn-1-palmitoyl-2-linoleoyl-PC and sn-1,2-dilinoleoyl-PC were reported in sunflower lecithin.

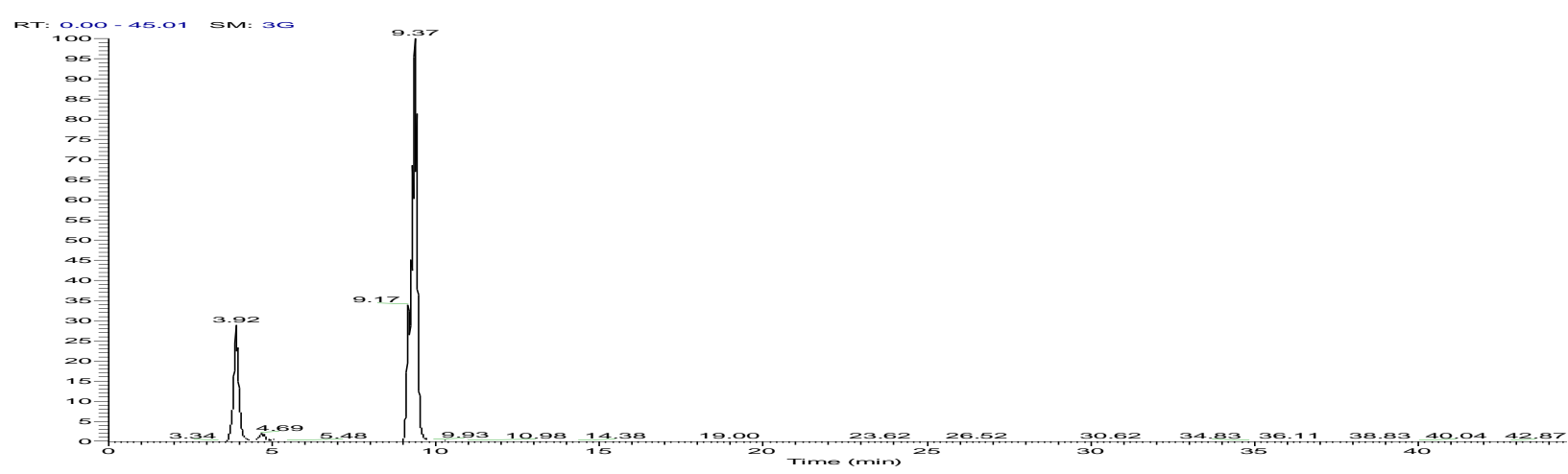




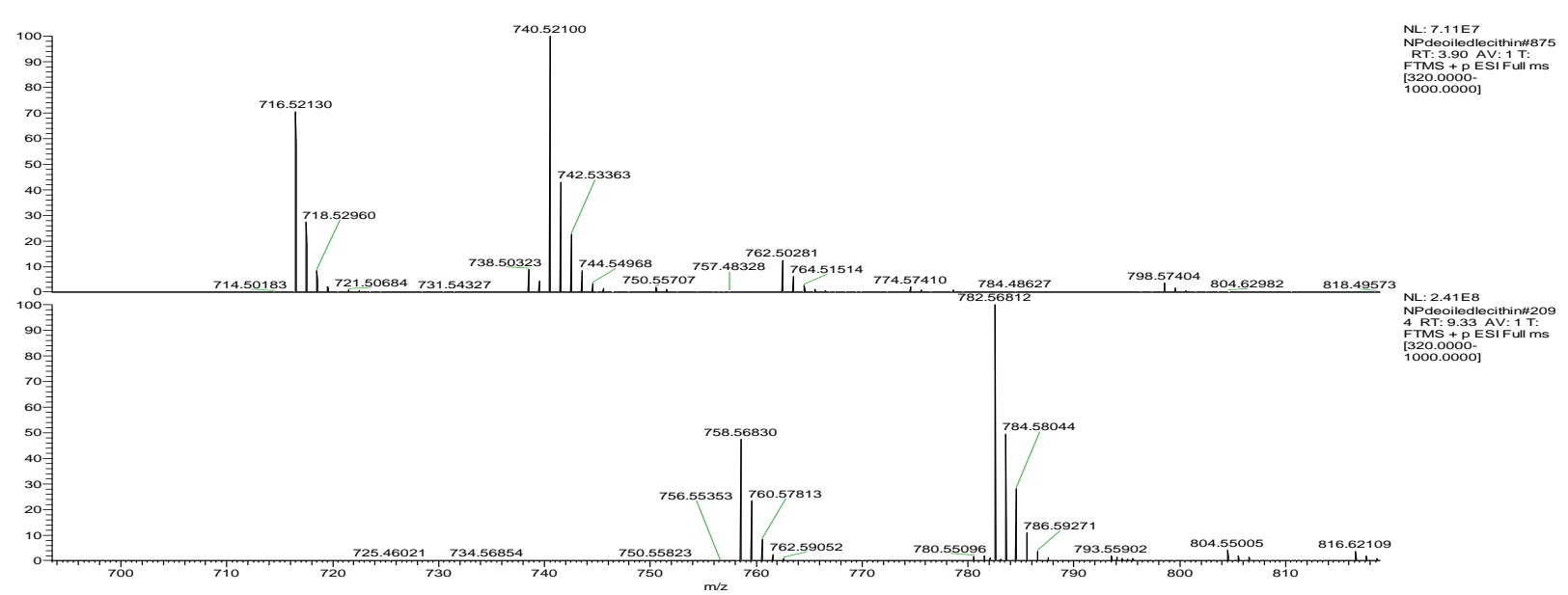

Figure 2. The chromatogram of de-oiled sunflower lecithin phospholipids by normal phase LC/MS (upper) and mass spectra of PE (RT=3.91 $\mathrm{min})$ and $\mathrm{PC}(\mathrm{RT}=9.37 \mathrm{~min})$ (lower)

With regard to the PE in de-oiled sunflower lecithin sample, the mass spectra showed the dominant species were $\mathrm{m} / \mathrm{z} 716.52130$ and $\mathrm{m} / \mathrm{z} 740.52100$. This indicated the presence of PE 34:2 and PE 36:4, as confirmed by their corresponding characteristic sodium adducts $[\mathrm{M}+\mathrm{Na}]^{+}$at $\mathrm{m} / \mathrm{z} 738.50323$ and $\mathrm{m} / \mathrm{z} 762.50281$. Since palmitic and linoleic acid are the main fatty acids in sunflower oil, it is reasonable to conclude that the PC and PE present in de-oiled sunflower lecithin samples were most likely esterified palmitic and linoleic acid.

To further separate different classes of PE and PC, reverse-phase high performance liquid chromatography was used to obtain their fingerprints from samples. Figure 3 shows the major PE and PC in de-oiled sunflower lecithin. As mentioned previously, PE 34:2 and PE 36:4 were the dominant PE species in de-oiled sunflower lecithin. In addition, PE 34:1 and PE 36:2 were also present in de-oiled sunflower lecithin. Similarly PC 34:2 and PC 36:4 were the major PC species in de-oiled sunflower lecithin with relative low amounts of PC 34:1 and PC $36: 2$.

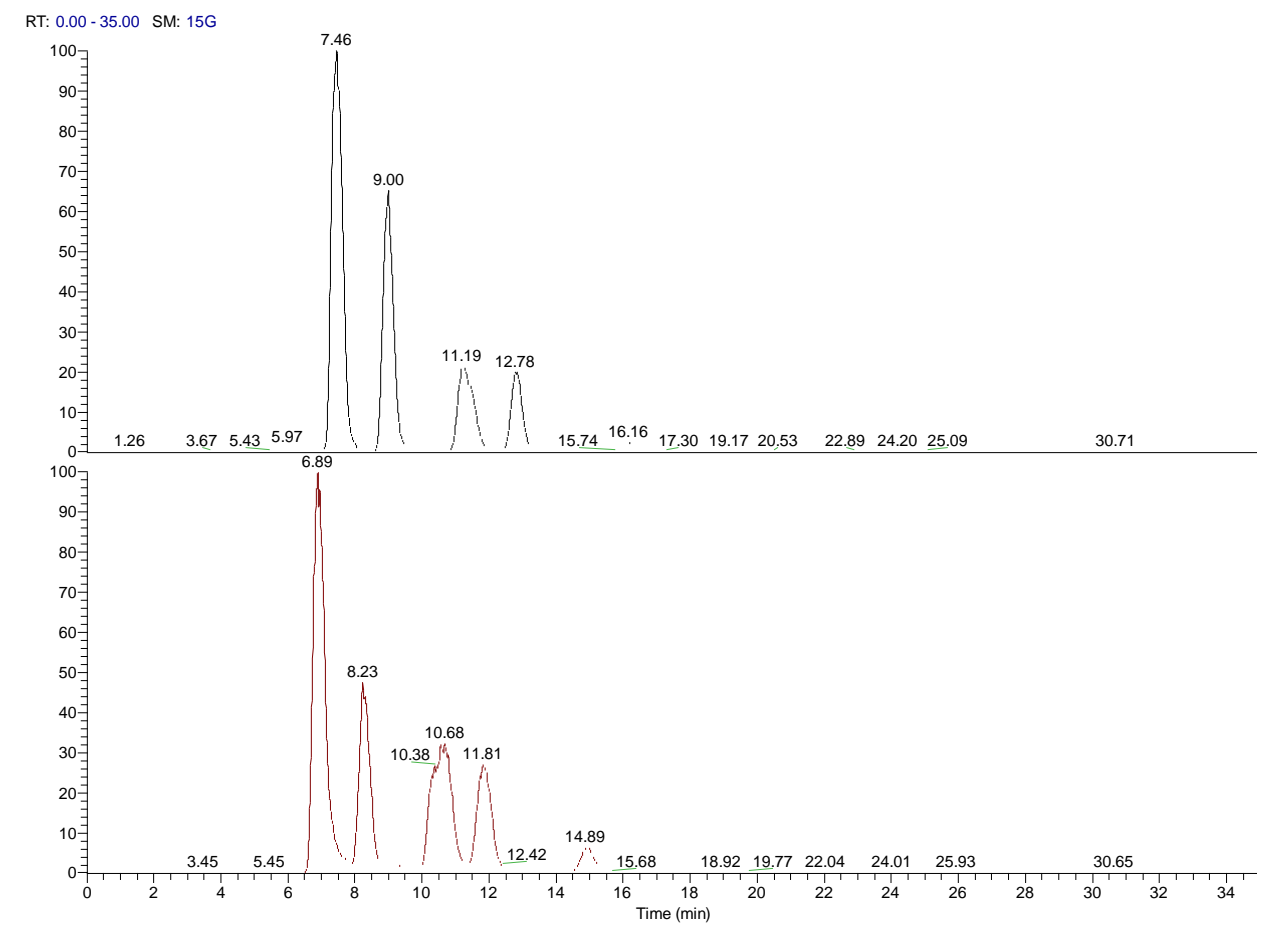

Figure 3. The chromatogram of PE (upper) and PC (lower) in de-oiled sunflower lecithin by reverse-phase LC/MS: PE 36:4 at 7.46min, PE 34:2 at 9min, PE 34:1 at 11.19min, PE 36:2 at 12.78min, PC 36:4 at 6.89min, PC $34: 2$ at $8.23 \mathrm{~min}, \mathrm{PC} 34: 1$ at $10.68 \mathrm{~min}$, and PC $36: 2$ at $11.81 \mathrm{~min}$ 
The four PE (PE 36:4, PE 34:2, PE 34:1 and PE 36:2) and four PC (PC 36:4, PC 34:2, PC 34:1 and PC 36:2) mentioned above were identified in freeze-dried and drum-dried apple powder (Fig. 4). PE 34:1 was not detected in freeze-dried apple powder but was detected in drum-dried apple powder, which is indicative of de-oiled sunflower lecithin phospholipid residue from the drum drying process. The PCs were all detected in both freeze-dried and drum-dried apple powder, except PC 34:1 was much lower in freeze-dried apple powder. It was discovered that the intensities of PE in freeze-dried and drum-dried apple powder were all approximately an order of magnitude lower than the respective PC intensities in those samples.
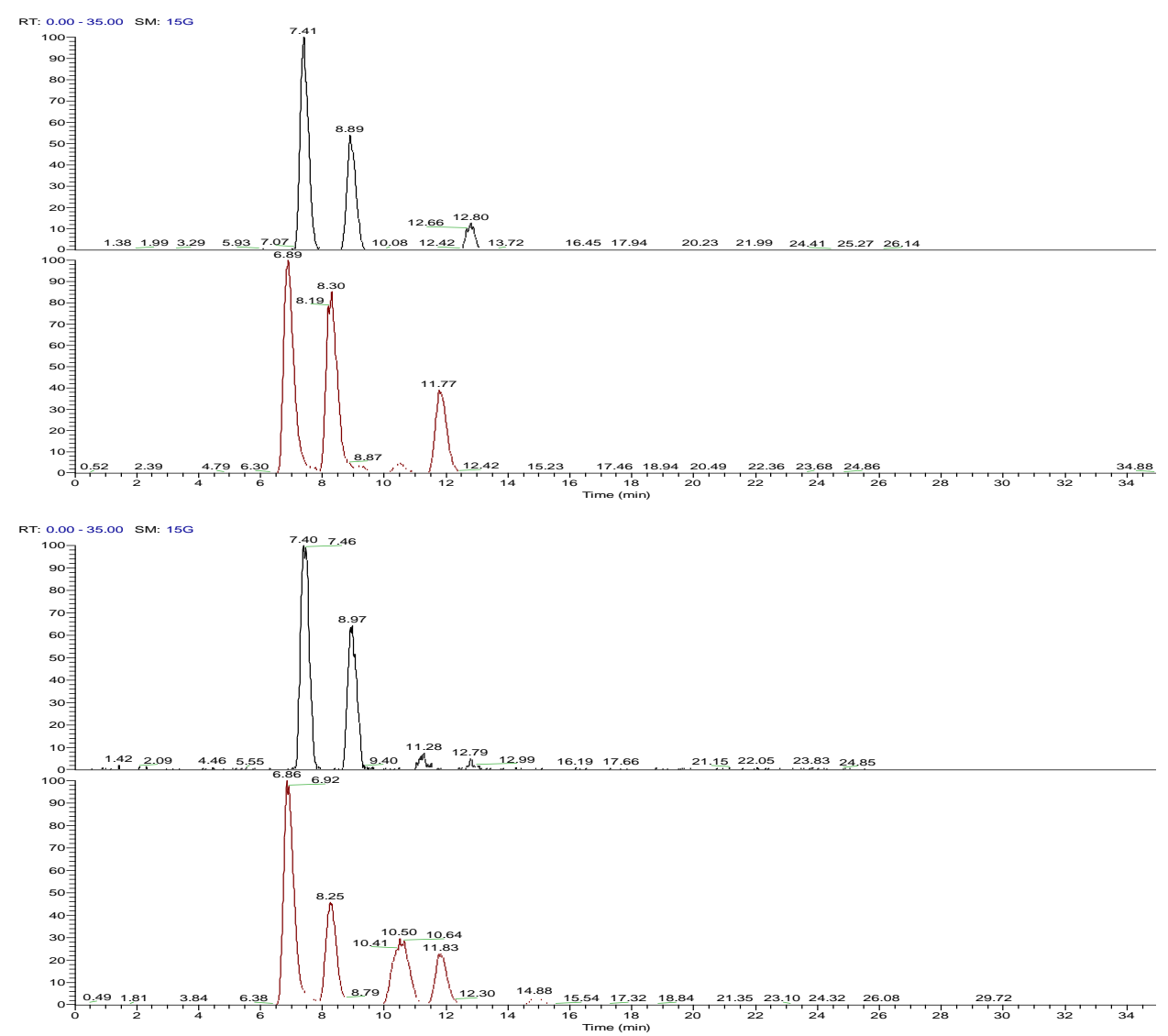

Figure 4. The chromatogram of PE and PC in freeze-dried and drum-dried apple powder by reverse-phase LC/MS. From the top to bottom of the figure, they are: PE profile (PE 36:4 at 7.41 min, PE 34:2 at 8.89min, PE $36: 2$ at $12.80 \mathrm{~min}$ ) in freeze-dried apple powder, PC profile (PC 36:4 at 6.89min, PC 34:2 at 8.30min, PC 34:1 at 10.50min, PC 36:2 at 11.77min) in freeze-dried apple powder, PE profile (PE 36:4 at 7.40min, PE 34:2 at 8.97min, PE 34:1 at 11.28min, PE 36:2 at 12.79min) in drum-dried apple powder and PC profile (PC 36:4 at 6.86min, PC 34:2 at 8.25min, PC 34:1 at $10.64 \mathrm{~min}$, PC 36:2 at 11.83min) in drum-dried apple powder

Similar to the profiles of apple powder, PE 36:4 and PE 34:2 were detected in all of the spinach powder samples. PE 36:2 was not found in freeze-dried spinach. Both PE 34:1 and PE 36:2 were not detected in drum-dried spinach. PC profiles of spinach powders were comparable to that of de-oiled sunflower lecithin, except with lower PC 36:2 in freeze-dried spinach. The PE intensity of spinach powder was noticeably lower than PC intensity.

Figure 5 shows the peak areas of the major phospholipids PE and PC in other fruits and vegetables. The PE and PC in each sample were identified by LC/MS. The peak area of different PE and PC was integrated to generate the bar chart for each sample. The vertical axle represents relative peak areas of four PE or PC in each sample. However, the bar charts cannot be used to quantitatively compare PE or PC among different samples. The profiles of PE from different drum-dried fruits are quite similar to PE profile of apple with dominant species of PE 34:2 and PE 36:4. No obvious PE 34:1 and PE 36:2 were detected in drum-dried and freeze-dried blueberry. PE 34:1 was the dominant PE in freeze-dried pineapple, cherry and broccoli seed. PE 34:2 was the major PE in freeze-dried sweet potato and pumpkin. 


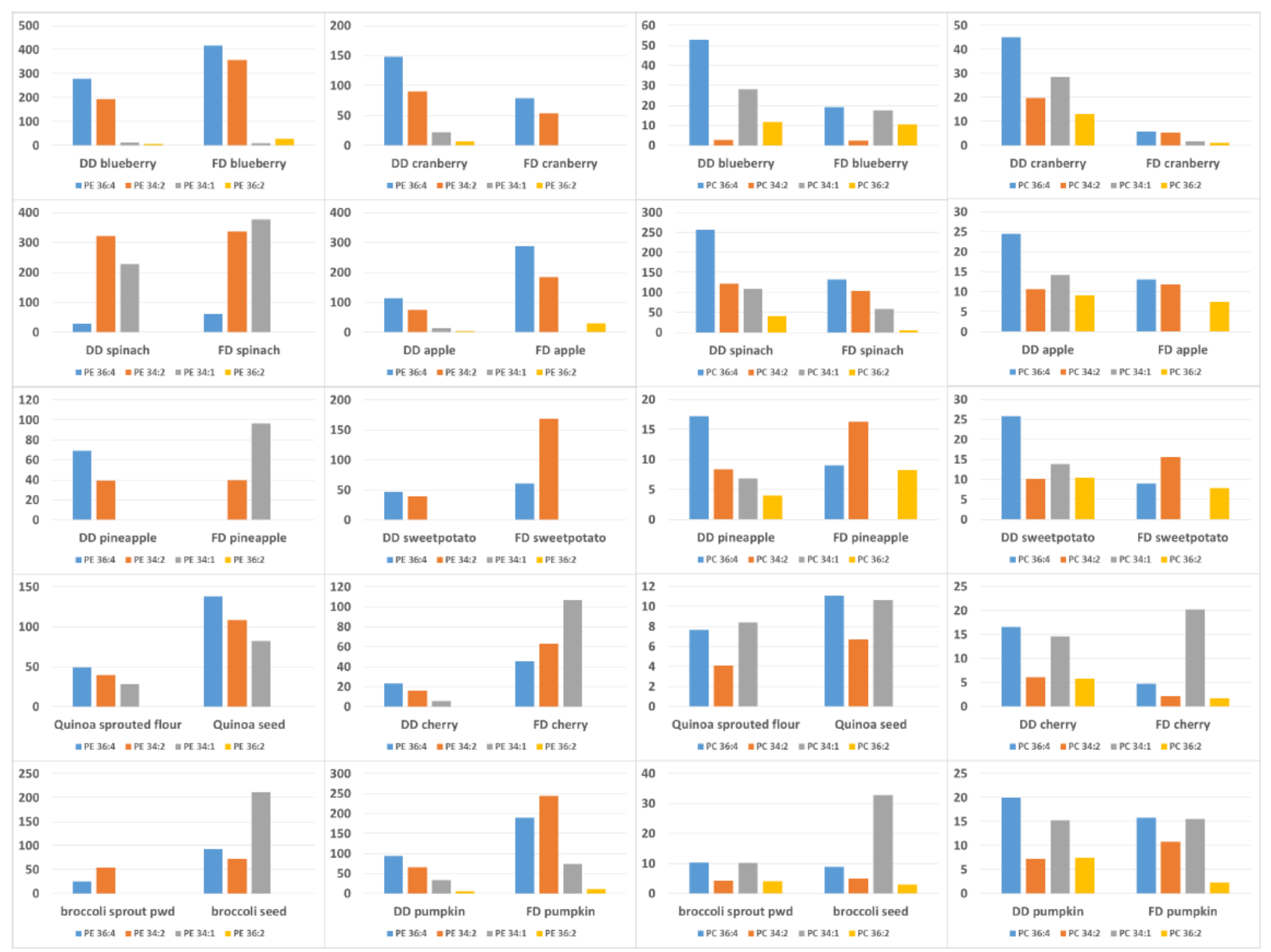

Figure 5. Peak areas of major phospholipids PE (PE 36:4, PE 34:2, PE 34:1, PE 36:2) and PC (PC 36:4, PC 34:2,

PC 34:1, PC 36:2) in different fruits and vegetables

The PC profiles of drum-dried product are comparable to the PC profile of de-oiled sunflower lecithin. The PC profile of freeze-dried blueberry was very different, with almost equal amounts of PC 34:2 and PC 34:1. PC 34:2 was the dominant PC in freeze-dried pineapple, and PC 34:1 was the major PC in cherry. No PC 34:1 was detected in freeze-dried sweet potato. No PC 36:2 was identified in quinoa sprout flour.

Since the content of PE in drum-dried product is far lower than the content of PC, it is reasonable to use the differences in PC concentration between drum-dried and freeze-dried product to estimate the residue of de-oiled sunflower lecithin phospholipid from drum drying process. However, this method should be used cautiously for other products in case the PE content is equal to or higher than PC content, or if other lipid classes are present. To test the proposed estimation method, the PE and PC profiles of various fruits and vegetables were examined. The peak area ratio of four major PE to four major PC for all of the samples analyzed is within 0.009 to 0.15 . As expected, the PE and PC profiles of different products are slightly different. This is because the fatty acid side chain present in PE and PC may vary with the product source of origin as different sources may provide a variety of fatty acids.

\subsection{Quantification of phosphatidylcholine in different natural products by HPLC-ELSD}

The effect of de-oiled sunflower lecithin phospholipids on final drum-dried product was subsequently investigated. Due to the detection limit of the ELSD detector, PS, PI and PE were unable to be quantified by ELSD in the samples studied. To quantify PC in the samples, individual PC compounds were used as external standards. Varying concentrations of standard solutions in the range from $35.6 \mu \mathrm{g} / \mathrm{mL}$ to $712 \mu \mathrm{g} / \mathrm{mL}$ were run before sample quantification analysis to obtain a calibration curve. Phospholipid content was then calculated and presented in Table 2. 
Table 2. Phosphatidylcholine in freeze-dried and drum-dried spinach, cranberry, cherry, blueberry, apple, pineapple, sweet potato, quinoa, pumpkin and broccoli sprout powder. ${ }^{\mathrm{a}}$

\begin{tabular}{lll}
\hline Sample Name & PC in drum-dried samples $(\mathrm{mg} / 100 \mathrm{~g})$ & PC in freeze-samples $(\mathrm{mg} / 100 \mathrm{~g})$ \\
\hline Spinach & $969.3 \pm 51.4$ & $748.0 \pm 155.5$ \\
Cranberry & $477.8 \pm 122.4$ & $<25.5^{\mathrm{b}}$ \\
Cherry & $148.6 \pm 2.2$ & $51.0 \pm 4.8$ \\
Blueberry & $202.1 \pm 37.5$ & $97.4 \pm 5.6$ \\
Apple & $214.8 \pm 10.0$ & $142.4 \pm 8.6$ \\
Pineapple & $<25.5^{\mathrm{b}}$ & $<25.5^{\mathrm{b}}$ \\
Sweet potato organic & $205.3 \pm 14.3$ & $94.5 \pm 14.0$ \\
Quinoa trim & $83.3 \pm 3.5$ & $139.4 \pm 7.1$ \\
pumpkin & $203.4 \pm 16.9$ & $<25.5^{\mathrm{b}}$ \\
broccoli sprout powder & $753.9 \pm 43.5$ & $489.3 \pm 26.8$ \\
${ }^{a}$ Values are averages \pm standard deviations & \\
was not detected in those samples by ELSD because the concentration was below detection limit. However,
\end{tabular}

PC in those samples were able to be identified by MS

The concentrations of PC in drum-dried and freeze-dried apple were higher than the concentrations of PC in the corresponding fresh products. Phosphatidylcholine in fresh apple has been reported by Wang and Faust (1992) with concentrations from $253.4 \pm 6.6 \mu \mathrm{g} / \mathrm{g}$ to $508.9 \pm 12.3 \mu \mathrm{g} / \mathrm{g}$. Picchioni et al. (Picchioni, Watada, Conway, Whitaker \& Sams, 1995) studied phospholipid composition of apple fruit and reported the concentration of PC varied from $161.4 \pm 7.3 \mathrm{mg} / 100 \mathrm{~g}$ to $131.4 \pm 4.8 \mathrm{mg} / 100 \mathrm{~g}$ dry wt depending on storage conditions. In this study, we investigated the phospholipids in apple powder for different drying processes. The PC concentration in freeze-dried apple was $142.4 \pm 8.6 \mathrm{mg} / 100 \mathrm{~g}$ dry $\mathrm{wt}$, and in drum-dried apple was $214.8 \pm 10.0 \mathrm{mg} / 100 \mathrm{~g}$ dry wt. The PC concentration in freeze dried apple was in close agreement with those reported by Picchioni et al. (1995, 1996) since lecithin was not added during the freeze-drying process; therefore, the phospholipid content of freeze-dried product should be close to the phospholipid content of fresh product.

The concentration of PC in spinach is even higher than the concentration of PC in apple. Previously, spinach was studied by Block et al. (1983) for purposes of comparing the lipid composition of the outer and inner envelop membranes. It was determined that a high amount of PC was present in the outer envelope membrane, whereas the inner envelope membrane had a polar lipid composition almost identical to that of thykaloids. No PE was detected in either envelop membrane. Edman and Ericson (1987) have studied the phospholipid composition in mitochondria from spinach leaves and petioles. Their results demonstrated that spinach leaf mitochondria contain relatively more PC than PE as compared to petiole mitochondria. These findings may potentially explain the higher PC concentration in spinach. In this study, both freeze-dried spinach powder and drum-dried spinach powder were analyzed. The PC concentrations in those samples were $748.0 \pm 155.5 \mathrm{mg} / 100 \mathrm{~g}$ and $969.3 \pm 51.4$ $\mathrm{mg} / 100 \mathrm{~g}$ respectively.

To further evaluate the effect of lecithin phospholipids in processed drum-dried products, PC in fresh cranberry, cherry, blueberry, pineapple, sweet potato, quinoa, and broccoli seeds and their corresponding drum-dried products were analyzed (Table 2). Similar to apple and spinach, PC in drum-dried cranberry, cherry, blueberry, sweet potato, pumpkin and broccoli sprout were higher than the PC in their corresponding fresh counterparts. Overall, the concentration of lecithin phospholipid residue in the drum dried powders we tested was less than $0.5 \%$. The analytical results from the products listed above demonstrate that adding de-oiled sunflower lecithin as a processing agent during drum drying does not affect the quality of final drum-dried product.

\section{Conclusion}

In this study, residues of sunflower lecithin phospholipids in drum-dried fruits and vegetables were investigated. The contents of phosphatidylcholine were quantified in spinach, apple, cranberry, cherry, blueberry, sweet potato, pineapple, quinoa trim, pumpkin and broccoli sprout as well as their residues after the drum drying process. Identification of phosphatidylcholine and phosphatidylethanolamine was conducted by normal-phase and reverse-phase ultra-high-performance liquid chromatography (UPLC) coupled with Q Exactive Orbitrap electrospray mass spectrometry. Quantification of phosphatidylcholine in various fruits and vegetables was performed using normal-phase high-performance liquid chromatography with evaporative light scattering detector (ELSD). Our novel methodological approach presented here could be used to evaluate de-oiled sunflower lecithin phospholipid residues in variety of drum-dried fruit and vegetable products. The developed 
HPLC-ELSD quantification method is economical and well-suited for quick estimation of lecithin phospholipid residues in drum-dried product. However, direct application for other species should be carefully considered due to different phospholipid profiles and potential interference from sample matrices.

\section{Acknowledgements}

Herewith we thank John Hunter (FutureCeuticals, Inc.) for review and providing valuable advice for editing this manuscript.

\section{References}

Aluyor, E. O., Ozigagu, C. E., Oboh, O. I., \& Aluyor, P. (2009). Chromatographic analysis of vegetable oils: A review. Scientific Research and Essay, 4(4), 191-197.

Becart, J., Chevalier, C., \& Blesse, J. P. (1990). Quantitative analysis of phospholipids by HPLC with a light scattering evaporating detector-application to raw materials for cosmetic use. Journal of high resolution chromatography, 13. 126-129. https://doi.org/10.1002/jhrc.1240130210

Block, M. A., Borne, A. J., Joyard, J., \& Douce, R. (1983). Preparation and characterization of membrane fractions enriched in outer and inner envelope membranes from spinach chloroplasts. The journal of biological chemistry, 258(21), 13281-13286. https://doi.org/10.1007/978-94-017-4973-2_5

Carelli, A. A., Brevedan, M. I. V., \& Crapiste, G. H. (1997). Quantitative determination of phospholipids in sunflower oil. J Amer Oil Chem Soc., 74, 511. https://doi.org/10.1007/s11746-997-0173-2.

Descalzo, A. M., Insani, E. M., \& Pensel, N. A. (2003). Light-scattering detection of phospholipids resolved by HPLC. Lipids, 38, 999. https://doi.org/10.1007/s11754-003-1154-1.

Dorne, A. J., Joyard, J., Block, M. A., \& Douce, R. (1985). Localization of phosphatidylcholine in outer envelope membrane of spinach chloroplasts. The journal of cell biology, 100, 1690-1697. https://doi.org/10.1007/978-94-017-4973-2_5

Edman, K., \& Ericson, I. (1987). Phospholipid and fatty acid composition in mitochondria from spinach (Spinacia oleracea) leaves and petioles. Biochem. J., 243, 575-578. https://doi.org/10.1042/bj2430575

Helmerich, G., \& Koehler, P. (2003). Comparison of methods for the quantitative determination of phospholipids in lecithins and flour improvers. Journal of agricultural and food chemistry, 51, 6645-6651. https://doi.org/10.1021/jf0345088

Jangle, R. D., Galge, R. V., Patil, V. V., \& Thorat, B. N. (2013). Selective HPLC method development for soy phosphatidylcholine fatty acids and its mass spectrometry. Indian journal of pharmaceutical sciences, 75(3), 339-345. https://doi.org/10.4103/0250-474x.117435

Jungalwala, F. B., Evans, J. E., \& McCluer, R. H. (1984). Compositional and molecular species analysis of phospholipids by high performance liquid chromatography coupled with chemical ionization mass spectrometry. Journal of lipid research, 25, 738-749.

Krüger, S., Bürmann, L., \& Morlock, G. E. (2015). Comparison and characterization of soybean and sunflower lecithins used for chocolate production by high-performance thin-layer chromatography with fluorescence detection and electrospray mass spectrometry. Journal of agricultural and food chemistry, 63, 2893-2901. https://doi.org/10.1021/jf506332f

Letter, W. S. (1992). A rapid method for phospholipid class separation by HPLC using an evaporative light-scattering detector. Journal of liquid chromatography, 15(2), 253-266. https://doi.org/10.1080/10826079208017168

McDonald, L. J., Robin, N. I., \& Siegel, L. (1981). New method for determining lecithin and sphingomyelin in amniotic fluid. Clin. Chem, 27/3, 410-416.

Picchioni, G. A., Watada, A. E., Conway, W. S., Whitaker, B. D., \& Sams, C. E. (1995). Phospholipid, galactolipid and steryl lipid composition of apple fruit cortical tissue following postharvest $\mathrm{CaCl} 2$ infiltration. Phytochemistry, 39(4), 763-769. https://doi.org/10.1016/0031-9422(95)00068-i

Picchioni, G. A., Watada, A. E., \& Whitaker, B. D. (1996). Quantitative high-performance liquid chromatography analysis of plant phospholipids and glycolipids using light-scattering detection. Lipids, 31(2), 217-221. https://doi.org/10.1007/bf02522623

Prabha, T. N., Raina, P. L., \& Patwardhan, M. V. (1988). Lipid profile of cultured cells of apple (Malus sylvestris) and apple tissue. J. Biosci, 13, 33-38. https://doi.org/10.1007/bf02832209 
Pua, C. K., Hamid, N. S. A., Rusul, G., \& Rahman, R. A. (2007). Production of drum-dried jackfruit (Artocarpus heterophyllus) powder with different concentration of soy lecithin and gum Arabic. Journal of food engineering, 78, 630-636. https://doi.org/10.1016/j.jfoodeng.2005.10.041

Robins, S. J., \& Patton, G. M. (1986). Separation of phospholipid molecular species by high performance liquid chromatography: potentials for use in metabolic studies. Journal of lipid research, 27, 131-139.

Sparace, S. A., \& Moore, T. S. (1979). Phospholipid metabolism in plant mitochondria. Plant physiol, 63, 963-972. https://doi.org/10.1104/pp.63.5.963

Stübiger, G., Pittenauer, E., Belgacem, O., Rehulka, P., Widhalm, K., \& Allmaier, G. (2009). Analysis of human plasma lipids and soybean lecithin by means of high-performance thin-layer chromatography and matrix-assisted laser desorption/ionization mass spectrometry. Rapid Commun. Mass Spectrom, 23, 2711-2723. https://doi.org/10.1002/rcm.4173

Tang, J., Feng, H., \& Shen, G. (2003). Drum drying. Encyclopedia of agricultural, food, and biological engineering, 211-214.

Wang, S. Y., \& Faust, M. (1992). Variation in lipid composition of apples in relation to watercore. J. Amer. Soc. Hort. Si., 117(5), 829-833.

\section{Copyrights}

Copyright for this article is retained by the author(s), with first publication rights granted to the journal.

This is an open-access article distributed under the terms and conditions of the Creative Commons Attribution license (http://creativecommons.org/licenses/by/4.0/). 